\title{
Archives
}

\section{Les débuts de la prise en charge de la gestion des documents administratifs au Centre intégré universitaire de santé et de services sociaux du Saguenay-Lac-Saint-Jean}

\section{DANY OUELLET}

Volume 47, numéro 2, 2018

URI : https://id.erudit.org/iderudit/1045166ar

DOI : https://doi.org/10.7202/1045166ar

Aller au sommaire du numéro

Éditeur(s)

Association des archivistes du Québec (AAQ)

ISSN

0044-9423 (imprimé)

2369-9256 (numérique)

Découvrir la revue

Citer cet article

OUELLET, D. (2018). Les débuts de la prise en charge de la gestion des documents administratifs au Centre intégré universitaire de santé et de services sociaux du Saguenay-Lac-Saint-Jean. Archives, 47(2), 21-41. https://doi.org/10.7202/1045166ar
Résumé de l'article

La mise en place d'un système de gestion intégrée des documents est un défi en soi, mais il est particulièrement ambitieux de le faire dans le contexte de fusion de structures aussi imposantes que celles des établissements du réseau de la santé. Dany Ouellet nous présente ici un retour sur l'expérience et les premières initiatives en ce sens au sein du Centre intégré universitaire de santé et de services sociaux du Saguenay-Lac-Saint-Jean. Le projet fut de taille en particulier par la diversité des approches de gestion documentaire et des outils en vigueur dans les différentes entités qui furent fusionnées suite à l'adoption par le gouvernement du Québec d'une loi à cet effet en 2015.

Dans cet article, l'auteur fait la recension des étapes parcourues jusqu'à maintenant et des problématiques soulevées. L'équipe de gestion documentaire nouvellement réunie dût d'abord constater les ressources disponibles, la masse de documents à traiter. Il fut nécessaire de tenir compte du respect des directives de Bibliothèque et Archives nationales du Québec, ainsi que des archives numériques, bien que les réalisations aient surtout été centrées sur les documents papier, dans un premier temps. L'auteur retient en conclusion que la reconnaissance de la gestion documentaire dans

l'organigramme de l'organisme et l'appui d'un gestionnaire dans un milieu où les archives administratives sont souvent négligées par rapport aux archives médicales, ont été des facteurs facilitant dans ce processus. Et il y a tout lieu d'être optimiste quant à la suite. 


\section{NOTES ET BILAN D'EXPÉRIENCE}

\section{Les débuts de la prise en charge de la gestion des documents administratifs au Centre intégré universitaire de santé et de services sociaux du Saguenay-Lac-Saint-Jean.}

\section{DANY OUELLET}

Archiviste-gestionnaire des documents, CIUSSS-SLSJ.

Consultant, Consuldoc enr., entreprise de services conseils et de support en gestion documentaire.

Chargé de cours, Université du Québec à Chicoutimi, certificat en gestion des documents et des archives.

\section{INTRODUCTION}

La Loi modifiant l'organisation et la gouvernance du réseau de la santé et des services sociaux notamment par l'abolition des agences régionales (aussi appelée Loi 10) est entrée officiellement en vigueur le $1^{\text {er }}$ avril 2015. Cette loi est à l'origine de la création des centres intégrés de la santé et des services sociaux du Québec (CISSS) et des centres intégrés universitaires de la santé et des services sociaux du Québec (CIUSSS), dont le Centre 
intégré universitaire de la santé et des services sociaux du SaguenayLac-Saint-Jean (CIUSSS-SLSJ).

Le CIUSSS-SLSJ est né de la fusion de neuf établissements eux-mêmes composés de plusieurs installations, dont les centres hospitaliers, les centres locaux de services communautaires, les centres hospitaliers de soins de longue durée et les différents points de services. ${ }^{1}$ Cette nouvelle entité regroupe près de 10000 employés répartis dans 60 installations et dispose d'un budget d'opération de plus de 786 millions de dollars. Ce réseau territorial de santé offre à 49 municipalités réparties sur $98710 \mathrm{~km}^{2}$ des services de santé physique, de santé mentale, de santé publique, des services généraux, du soutien à l'autonomie des personnes âgées, aux jeunes en difficulté, ainsi que dans les domaines de la déficience physique, de la déficience intellectuelle/trouble du spectre de l'autisme et de la dépendance. ${ }^{2}$

Outre ce changement majeur dans la structure organisationnelle du réseau régional de la santé et des services sociaux, la loi rappelle aux instances concernées du CIUSSS-SLSJ leurs obligations légales en matière de prise en charge des documents hérités des neuf établissements fusionnés. Ainsi, l'article 202 stipule que:

Les dossiers et les documents d'un établissement fusionné et d'une agence de la santé et des services sociaux deviennent, sans autre formalité, les dossiers et documents du centre intégré de santé et de services sociaux qui leur succède.

De plus, les dossiers des usagers d'un établissement regroupé sont réputés être aussi les dossiers des usagers du centre intégré dont le conseil d'administration administre l'établissement regroupé. (Loi 10, 2015, p. 49)

Conséquemment, le CIUSSS-SLSJ devient responsable, non seulement de ses propres documents administratifs et cliniques qu'il produit et reçoit depuis sa création, mais aussi de la conservation et de l'accessibilité des documents hérités de tous les établissements fusionnés, incluant les documents en formats papier et numérique actifs, semi-actifs et les divers fonds d'archives historiques. Par ailleurs, nous retrouvons parmi les documents historiques différents supports: vidéocassettes, cassettes sonores, diapositives, plaques commémoratives, sceaux, etc. 
L'objectif du présent article est, d'une part, de présenter les premières initiatives entreprises dans un établissement de santé en vue d'implanter un système de gestion intégrée des documents administratifs (SGID), soit le CIUSSS-SLSJ. Dans le cadre de cet article, nous définissons le SGID comme étant l'ensemble des outils et des opérations qui permet de gérer tous les documents administratifs produits et reçus par un établissement à l'aide d'un unique logiciel spécialisé.

D'autre part, nous espérons que cette expérience inspirera une démarche concrète de gestion des documents administratifs pour les autres établissements du réseau de la santé et des services sociaux de la province qui n'ont pas encore entrepris une telle initiative. ${ }^{3}$ Finalement, il serait souhaitable qu'une véritable et fructueuse collaboration s'installe peu à peu entre les responsables de la gestion documentaire des différents établissements du réseau de la santé.

\section{LES PREMIERS PAS}

Les changements structurels majeurs issus de la loi précitée interpellaient directement les personnes responsables de la gestion des documents administratifs au sein d'un établissement fusionné. Qu'allait-il se passer en cette matière? Les calendriers de conservation des établissements fusionnés s'appliqueraient-ils toujours? Qui serait responsable du traitement des fonds d'archives historiques hérités par chaque établissement fusionné? Comment arrimerait-on les outils de gestion des documents administratifs de chacun? Quelle serait la position de la "gestion documentaire» vis-à-vis de la gestion des archives médicales sur l'échiquier de la gestion de l'information documentaire dans le futur CIUSSS-SLSJ ? Qui ? Quoi ? Comment? Où?... Bref, le $1^{\text {er }}$ avril 2015... beaucoup de questions en suspens et de matière à réflexion.

\subsection{Une initiative personnelle à l'échelle provinciale}

En septembre 2014, à la suite du dépôt du projet de loi 10, la fusion était imminente. Nous avons alors pris l'initiative de livrer nos réflexions sur la problématique de la gestion des documents administratifs dans le réseau de la santé à plusieurs acteurs concernés par notre champ $\mathrm{d}^{\prime}$ activité professionnelle ${ }^{4}$. 
En nous inspirant d'un article de Bouchard, Gendron et Ducasse, paru dans la revue Archives en 1992, qui traçait un état de situation de la gestion des documents administratifs dans les établissements de santé et de services sociaux de l'époque au Québec, et en nous basant sur une enquête personnelle réalisée dans les centres de santé et de services sociaux (CSSS) du Québec en 2006, nous concluions notre rapport de novembre 2014, intitulé La réforme de notre système de santé et la gestion de l'information documentaire, de la façon suivante:

Serait déconnecté qui oserait prétendre que la gestion des documents et l'application rigoureuse des articles de lois susmentionnées sont des priorités à l'intérieur du plan d'action de l'actuelle réforme de notre réseau de santé. Cependant, outre les archives médicales, il y a une masse de documents administratifs dans nos établissements de santé et des lois qui encadrent leur création, leur utilisation et leur disposition. Nous ne pouvons I'ignorer.

Ne serait-ce pas le moment idéal pour certaines organisations phares en gestion de l'information documentaire au Québec d'agir, de faire valoir la nécessité légale et les avantages pratiques de voir à la bonne gestion de la masse documentaire administrative que l'on retrouve dans les établissements de santé et le futur CISSS? (Ouellet, 2014)

On ne peut, actuellement, mesurer avec certitude les résultats de cette démarche individuelle ni faire un lien avec les écrits du Comité national des archives de la santé et des services sociaux (CNASSS ${ }^{5}$ ). Toutefois, nous sentions la nécessité de poursuivre notre effort de sensibilisation.

\subsection{Des actions professionnelles au niveau régional}

Lorsque la fusion fut officialisée, nous avons déposé en mai 2015, auprès de nos nouveaux supérieurs, un document intitulé La prise en charge de la gestion documentaire au Centre intégré universitaire de santé et des services sociaux (CIUSSS) du Saguenay-Lac-Saint-Jean: une nécessité. Dès le mois d'octobre 2015, nous avons rencontré ces derniers afin de discuter de ce document. Nous leurs avons rappelé, entre autres choses, les avantages pratiques et les obligations légales relatives à la 
gestion des documents administratifs pour le CIUSSS-SLSJ, à l'instar des autres organisations du secteur public.

Ainsi, le CIUSSS-SLSJ doit adopter une politique de gestion de ses documents actifs et semi-actifs (article 6 de la Loi sur les archives). II doit aussi assumer la gestion de ses documents historiques, incluant ceux des établissements fusionnés (articles 15, 17 et 18 de la Loi sur les archives). À cet effet, une directive de BAnQ de mai 2015 précise qu'avec la fusion, les fonds d'archives des établissements fusionnés sont fermés. Les calendriers de conservation de ces établissements ne s'appliquent que pour les documents inactifs avant le $1^{\text {er }}$ avril 2015. Le CIUSSS-SLSJ doit donc établir, faire approuver, tenir à jour et appliquer un nouveau calendrier de conservation pour les documents actifs produits à partir du $1^{\text {er }}$ avril 2015 et pour les documents toujours semi-actifs produits par les établissements fusionnés au $1^{\text {er }}$ avril 2015 (articles 7 et 8 de la Loi sur les archives).

Par ailleurs, notre établissement doit faciliter l'accès à ses documents, entre autres par la création d'un plan de classification (articles 9 et 16 de la Loi sur l'accès aux documents des organismes publics et sur la protection des renseignements personnels). Finalement, le CIUSSS-SLSJ est dans l'obligation de concevoir et de maintenir à jour un inventaire des fichiers de renseignements personnels qu'il détient (article 76 de la Loi sur l'accès aux documents des organismes publics et sur la protection des renseignements personnels).

L'objectif de cette démarche à l'interne visait à rappeler la nécessité pour le CIUSSS-SLSJ de voir à la conception, à l'implantation physique et au suivi d'un SGID digne de ce nom, c'est-à-dire fonctionnel à court et moyen termes, et durable à long terme. Sur ce, il fallait donc insister, d'une part, sur l'importance d'inscrire la gestion des documents administratifs dans l'organigramme de l'établissement et, d'autre part, sur la nécessité de nommer un gestionnaire responsable de la gestion des documents administratifs et de former un comité qui prendra en charge la gestion documentaire.

Cette démarche s'est avérée fructueuse puisque, au mois d'octobre 2015, la "Gestion documentaire» était inscrite dans l'organigramme du CIUSSS-SLSJ et rattachée à la «Direction des ressources humaines, communications et affaires juridiques». De plus, la «Chef de services Affaires juridiques » devenait officiellement responsable de ce dossier et du futur comité de gestion des documents administratifs. 


\subsection{Le Comité national des archives de la santé et des services sociaux (CNASSS)}

Parallèlement à nos actions, en janvier 2015, le CNASSS a distribué dans le réseau de la santé le document intitulé Les documents inactifs du réseau de la santé et des services sociaux dans le contexte de la loi 10: principes directeurs ${ }^{6}$.

Ce document avait pour but de sensibiliser les responsables des établissements de santé et de services sociaux à leurs obligations légales envers leurs documents et leurs archives dans le contexte de la restructuration des services. On y précise la manière de gérer les documents actifs, semi-actifs et inactifs des établissements fusionnés, et rappelle l'obligation, pour le CIUSSS, d'élaborer et de faire approuver son propre calendrier de conservation.

Cette initiative a eu un écho au CIUSSS-SLSJ. En effet, en mai 2015, les autorités concernées de notre établissement ont reçu un communiqué de BAnQ résumant les principaux points soulevés dans le document susmentionné. Selon nous, cette démarche du CNASSS est importante dans la mesure où elle a vraisemblablement amené chez nos directeurs une plus grande sensibilisation à la nécessaire prise en charge de la gestion des documents administratifs dans notre établissement.

\section{L'ÉQUIPE DE GESTION DOCUMENTAIRE}

Avant la création du CIUSSS-SLSJ, quatre personnes avaient des responsabilités en gestion des documents administratifs dans leur établissement: une technicienne en documentation (Agence de la santé et des services sociaux); un archiviste (Centre jeunesse); une spécialiste en procédés administratifs (Centre de réadaptation en déficience intellectuelle et en troubles envahissants du développement); un spécialiste en procédés administratifs (CSSS Domaine-du-Roy).

Jusqu'alors, nous n'avions pas de comité régional ni même de rencontres informelles entre nous. Chacun travaillait, en quelque sorte, isolément dans son établissement. Toutefois, avec la venue du nouvel établissement, ces ressources ont été identifiées afin de former le comité responsable de la gestion des documents administratifs. La première 
rencontre formelle de l' "Équipe de gestion documentaire » a eu lieu le 18 novembre 2015. Nous y avons principalement défini notre mandat et établi un premier plan d'action.

À ce jour, nos rencontres sont régulières et nos échanges sont facilités par la création d'un espace réseau électronique réservé à l'équipe. Par ailleurs, il est important de mentionner que chaque membre de l'équipe conserve toujours ses responsabilités rattachées à son établissement fusionné, en sus de sa participation à l'intérieur de l'équipe, période de transition oblige. Finalement, de façon à faciliter notre travail sur le terrain à l'intérieur de notre vaste territoire régional, chacun devient répondant pour un certain nombre d'installations.

\subsection{Le mandat}

À l'instar de tout groupe de travail ayant la responsabilité de la gestion des documents administratifs dans son organisation, le mandat de notre équipe de gestion documentaire est de nous doter d'une gouvernance documentaire efficiente, entre autres de planifier, d'implanter, d'administrer et d'évaluer un SGID en tenant compte de la capacité et des moyens de l'établissement.

\subsection{Le premier Plan d'action (2015)}

Notre équipe a préparé un plan d'action qui se voulait général au départ. Celui-ci a trois objectifs:

1. Démontrer aux gestionnaires la nécessité de mettre en place un SGID pour répondre aux obligations légales et aux besoins administratifs de repérage, d'accès et de conservation de l'information;

2. Présenter le mandat de l'équipe de gestion documentaire;

3. Proposer un schéma d'action sous forme d'une structure de gestion de projet avec des objectifs, des moyens, des outils et des échéanciers.

Ce plan d'action a été présenté au comité de la haute direction du CIUSSS-SLSJ ${ }^{7}$ en décembre 2015. 


\subsection{Le second Plan d'action (2016)}

Notre équipe a été assez tôt confrontée à la réalité. De fait, les démarches amorcées afin de concevoir les outils de gestion des documents administratifs (plan de classification, calendrier de conservation, logiciel de gestion intégrée des documents) ont été ralenties pour laisser place à d'autres priorités. Une grande partie de nos efforts ont, entre autres, porté sur la prise en charge de la masse documentaire (les documents semi-actifs et les archives historiques) laissée pour compte et résultant des nombreux déménagements de personnel et des restructurations. Ce contexte a forcé l'équipe de gestion documentaire à préciser son premier plan d'action.

Le second plan d'action d'octobre 2016 vise donc le court terme, soit la période d'octobre 2016 à avril 2017. II s'oriente sur trois axes:

1. Faire connaître le rôle de l'équipe de gestion documentaire à I'ensemble du CIUSSS-SLSJ;

2. Rechercher les espaces physiques requis pour y regrouper et inventorier sommairement les documents inactifs retrouvés dans chacun des neuf établissements fusionnés;

3. Poursuivre la production des outils de gestion des documents administratifs.

\subsection{Les premières réalisations (2015-2016)}

À l'intérieur du plan d'action de décembre 2015, notre équipe propose un projet de gestion des documents administratifs portant sur cinq livrables, soit "Le portrait documentaire de l'établissement » (livrable 1), "La conception des outils de gestion des documents administratifs » (livrable 2), "L'implantation physique du SGID» (livrable 3), "L'administration du SGID» (livrable 4) et «L'évaluation du SGID » (livrable 5).

\subsubsection{Livrable 1: Le portrait documentaire de l'établissement}

L'objectif premier de cet exercice était de produire un portrait aussi fidèle et complet que possible de la gestion des documents administratifs dans l'ensemble des établissements fusionnés. Entre autres, il s'avérait fort 
intéressant d'évaluer la masse documentaire active et inactive présente dans l'ensemble de ces établissements depuis leur création. Dans certains cas, cela remonte à près d'un siècle. Afin de réaliser ce premier mandat, notre équipe a d'abord conçu un guide de prise d'inventaire. ${ }^{8}$

En nous appuyant sur ce guide, nous avons ensuite effectué une série de visites et de contacts téléphoniques auprès de différents secteurs d'activités de chaque établissement fusionné. Cette démarche s'est échelonnée du 30 décembre 2015 au 14 juin 2016. Elle a fait l'objet d'un rapport intitulé État de situation de la gestion des documents administratifs des neuf établissements fusionnés. Ce document a été déposé au comité de la haute direction du CIUSSS-SLSJ en octobre 2016.

Ce portrait fait état de plusieurs éléments fort intéressants. Ainsi :

A. Évaluation sommaire de l'usage des outils de gestion des documents administratifs

- 7 établissements sur 9 (78 \%) n'avaient aucune politique de gestion des documents administratifs à jour.

- 6 établissements sur 9 (61 \%) n'avaient pas de plan de classification organisationnel à jour ou n'en n'avaient tout simplement pas.

- 1 seul établissement sur 9 (11\%) possédait un calendrier de conservation à jour.

- 7 établissements sur 9 (78\%) possédaient un des cinq logiciels de gestion intégrée des documents (ci-après: GID) utilisés dans la région ${ }^{9}$. Toutefois, pour 1 seul (14\%), la base de données du logiciel était à jour et utilisée par la majorité de ses grandes directions. Pour les 6 autres (86\%), le logiciel n'était utilisé qu'occasionnellement et un système secondaire basé sur des listes produites par un logiciel de bureautique était opérationnel.

- 2 établissements sur 9 (22\%) possédaient un guide de gestion des documents administratifs.

- 6 établissements sur 9 (67\%) n'avaient pas de liste de documents essentiels disponible ni de mesures de protection sur ce plan. 
- 9 établissements sur $9(100 \%)$ se disaient sensibilisés à la confidentialité et la protection des renseignements personnels. Cependant, nulle part on ne pouvait identifier systématiquement l'ensemble de ces documents. De plus, un seul établissement $(11 \%)$ avait élaboré un projet d'inventaire des fichiers de renseignements personnels, tel qu'exigé par la Loi sur l'accès aux documents des organismes publics et sur la protection des renseignements personnels.

- Aucun établissement (0 \%) ne possédait un plan de classification organisationnel pour les documents numériques (arborescence uniforme) ni de règles de nommage des répertoires et fichiers numériques. Chaque producteur/utilisateur avait son propre classement sur son poste de travail ou partagé en réseau.

B. Évaluation sommaire de la masse documentaire

Les résultats suivants présentent la volumétrie documentaire connue et inventoriée dans les différents établissements fusionnés. Compte tenu de certains secteurs d'activités qui n'ont pas été rencontrés, ils sont donc partiels et constituent, à notre avis, un minimum.

\begin{tabular}{|c|c|}
\hline État de conservation & $\begin{array}{c}\text { Quantité de documents } \\
\text { en mètres linéaires }\end{array}$ \\
\hline Documents actifs & 1400 mètres ou 1,4 kilomètre \\
\hline Documents semi-actifs & 1700 mètres ou 1,7 kilomètre \\
\hline Documents inactifs & 1300 mètres ou 1,3 kilomètre \\
\hline TOTAL: & 4400 mètres ou 4,4 kilomètres \\
\hline
\end{tabular}

Tableau 1: Masse documentaire des 9 établissements fusionnés ${ }^{10}$

\section{Conclusion et recommandations}

Ces résultats montrent une gestion documentaire très fragmentaire et hétérogène d'un établissement à un autre. Certes, certains outils de gestion des documents administratifs étaient mis en place, mais peu d'entre eux étaient utilisés à l'échelle de l'organisation à l'intérieur d'une approche 
systémique de la gestion des documents administratifs et dans le cadre d'un véritable programme de gestion des documents administratifs. Bref, nous sommes loin d'un système de gestion des documents administratifs digne de ce nom.

Devant ces constats, l'équipe de gestion documentaire a établi trois niveaux de priorités d'actions. Les priorités 1 consistent à faire connaître la gestion intégrée des documents à l'ensemble de l'organisation, puis à faire reconnaître le rôleclé des agentes administratives (secrétaires, techniciennes, etc.) dans la gestion quotidienne du futur SGID. Les priorités 2 sont, d'abord, de prévoir des espaces, des conditions d'entreposage et du matériel adéquats afin d'accueillir la masse de documents inactifs hérités des établissements fusionnés. Ensuite, il s'agit d'effectuer un inventaire, au moins sommaire, de l'importante quantité de documents identifiée. Enfin, les priorités 3 visent, d'une part, à poursuivre la conception du plan de classification et du calendrier de conservation et, d'autre part, à poursuivre les démarches en vue d'acquérir un logiciel de GID qui supportera I'implantation de notre SGID. (CIUSSS, Équipe de gestion documentaire, 2016)

\subsubsection{Livrable 2: La conception des outils de gestion des documents administratifs}

La création du CIUSSS-SLSJ obligeait notre équipe à concevoir de nouveaux outils de gestion des documents administratifs. Dans la mesure du possible, nous désirions nous inspirer des outils déjà utilisés par les établissements fusionnés.

\subsubsection{La politique de gestion des documents administratifs}

En plus d'être une obligation légale de par la Loi sur les archives, cet outil reflète la volonté de l'organisation de prendre en charge la gestion des documents administratifs et en définit les responsabilités. De fait, nous sommes d'avis que l'adoption de notre Politique relative à la gestion des documents administratifs, datant de juin 2016, constitue la pierre d'assise du futur SGID. De plus, elle représente un levier important soutenant nos démarches en vue de réaliser les objectifs définis à l'intérieur de nos plans d'action. 
Ainsi, les objectifs de cette politique sont d'abord de favoriser la prise en charge efficace de l'ensemble des documents administratifs par la conception, l'implantation, le suivi et l'évaluation d'un système de gestion des documents administratifs uniforme, fonctionnel et durable. Ensuite, ils visent à soutenir l'utilisation d'outils appropriés pour optimiser la gestion des documents administratifs. Finalement, ils permettent à notre établissement de mettre en place les mesures nécessaires pour assurer le contrôle, l'intégrité, la sauvegarde, l'accès et la confidentialité des documents administratifs tout au long de leur cycle de vie.

Cette politique concerne les documents administratifs, quels qu'en soient la nature, le support sur lequel ils sont fixés, leur état de conservation et leur localisation ${ }^{11}$.

Finalement, des responsabilités sont définies pour le comité de la haute direction, pour la Direction des ressources humaines, pour le service des Communications et affaires juridiques, pour la Direction des ressources informationnelles (service informatique), pour l'ensemble des autres directions, pour le personnel de soutien administratif, pour les utilisateurs du système de gestion des documents administratifs et, bien sûr, pour l'équipe de gestion documentaire.

Afin de remplir adéquatement son mandat, notre équipe doit donc s'assurer de la bonne gouvernance du système de gestion des documents administratifs ${ }^{12}$, prendre en charge la création, la mise à jour et l'application des outils de gestion des documents administratifs, supporter les directions et le personnel de soutien administratif concernés dans la gestion efficace des documents actifs sous leur gouverne et voir à la bonne gestion des documents semi-actifs et des archives historiques.

\subsubsection{Le plan de classification et le calendrier de conservation}

Notre équipe a décidé dès le départ de concevoir parallèlement ces deux outils de gestion des documents administratifs. Pour ce faire, nous nous sommes inspirés du guide fourni par le CNASSSS, soit le Recueil des règles de conservation des documents des établissements de santé et de services sociaux du Québec.

Nos travaux ont débuté en mai 2015 et se poursuivent toujours. La partie concernant les séries de gestion a été complétée en novembre 2016. 
Il est prévu que la saisie s'effectue via le système transactionnel de BAnQ, $G_{A L A}{ }^{13}$. Nous complèterons le plan de classification et le calendrier de conservation au fur et à mesure que le CNASSS rendra disponible les règles relatives aux autres séries documentaires de mission.

\subsubsection{Le choix d'un logiciel de gestion intégrée des documents}

Le logiciel de gestion intégrée des documents est l'élément fédérateur d'un SGID, en ce sens qu'il intègre le plan de classification, le calendrier de conservation et constitue l'outil principal de repérage pour l'ensemble des documents administratifs en formats papier et numérique.

L'état de situation, déposé en octobre 2016, a montré que cinq logiciels différents étaient et sont toujours utilisés à différents degrés par certains établissements fusionnés. II est donc prioritaire de procéder à l'acquisition d'une seule application qui sera disponible pour l'ensemble des directions de notre établissement. Avec la fusion, les fonds d'archives des anciens établissements doivent être fermés. Afin d'assurer l'intégrité du fonds du CIUSSS-SLSJ, cela nous oblige à migrer les informations contenues dans les banques de données de ces logiciels.

Dès mars 2015, nous avons entamé nos réflexions et nos discussions sur le dossier. Cependant, en avril, nous étions informés de l'existence d'un moratoire sur tout projet d'achat de logiciel. Ce moratoire concerne-t-il le renouvellement des licences? Le choix d'un logiciel parmi les logiciels de GID déjà en place dans notre établissement est-il soumis au moratoire? Malgré l'incertitude qui planait, nous avons préparé, en collaboration avec le service informatique et en nous s'inspirant de grilles d'évaluation glanées ici et là parmi nos collègues archivistes du réseau, un plan de démarrage de projet pour l'acquisition d'un logiciel de GID en juillet 2015. Toutefois, un changement de porteur de dossier sur le plan informatique et les nombreux autres projets informatiques en cours ont fait en sorte qu'en décembre 2016 le dossier tardait toujours à débloquer. Nous avons néanmoins eu l'autorisation de renouveler pour une période limitée les licences des logiciels déjà installés dans les établissements fusionnés ${ }^{14}$. En janvier 2017, l'équipe de gestion documentaire a réactivé le dossier en consacrant ses efforts à l'analyse détaillée de quelques solutions logicielles existantes. Notre objectif est de choisir une application au cours de la présente année. 


\subsection{Les autres problématiques}

Au moment d'écrire cet article, le CIUSSS-SLSJ a seulement quelques mois d'existence. Tel que mentionné précédemment, au cours de cette période de démarrage, notre équipe de gestion documentaire a dû réorienter ses interventions en fonction de la réalité du terrain. Ainsi, outre nos actions précédentes devant conduire à l'implantation d'un SGID, certaines problématiques nous ont particulièrement interpellés.

\subsubsection{Le déplacement de personnel}

La période de transition actuelle provoque un important remaniement organisationnel. Celui-ci entraîne de nombreux changements de poste et de déménagements internes de direction et d'individus d'une installation physique à une autre. Cette situation nous permet de constater, en premier lieu, que certaines personnes apportent avec elles des dossiers actifs en format papier et laissent sur place une quantité non négligeable de dossiers inactifs (semi-actifs et archives historiques). En second lieu, nous avons remarqué que quelques employés décident d'épurer euxmêmes leurs dossiers papier avant de quitter leur poste. Dans l'ensemble, il appert finalement que peu d'employés semblent préoccupés par le traitement de leurs documents en format numérique.

De décembre 2015 à décembre 2016, notre équipe de gestion documentaire a donc été dans l'obligation d'intervenir à plusieurs reprises afin de conserver un certain contrôle de la masse documentaire devenue « orpheline». Ainsi, nous avons d'abord pris l'initiative de distribuer deux notes de service. La première (décembre 2015) était adressée à tous les employés et leur indiquait la marche à suivre générale en ce qui concerne la gestion de leurs documents actifs et inactifs en formats papier et numérique. La seconde (janvier 2016) fut distribuée aux gestionnaires et leur proposait un mécanisme de soutien à leurs équipes lors de la préparation de dossiers, à classer ou à déménager, de façon à assurer la sécurité, l'intégrité et la confidentialité des informations.

Dans la foulée de l'envoi de ces directives, nous avons préparé une procédure de transfert des dossiers inactifs pour faciliter l'application de ces notes: épuration, inventaire sommaire, emboîtage, déménagement et entreposage. En parallèle, nous avons soutenu à plusieurs occasions des employés qui désiraient faire du ménage dans leurs dossiers inactifs. 
Pour ce faire, en collaboration avec BAnQ, nous avons préalablement récupéré les calendriers de conservation des établissements fusionnés. Cela nous a permis de répondre à certaines questions précises en regard des règles de conservation légalement applicables et de nous conformer aux directives de BAnQ en la matière.

Ensuite, nous devrons nous occuper prioritairement de la quantité importante de documents inactifs laissés sur place, et ce, dans de brefs délais. En effet, à plusieurs endroits, on procède au réaménagement physique de locaux qui doivent être réoccupés par le personnel. II devient, en quelque sorte, urgent d'inventorier le contenu des boîtes et de trouver rapidement des locaux pour les accueillir. II y a un risque bien présent que bon nombre de dossiers deviennent introuvables s'ils sont déplacés à l'insu de l'équipe de gestion documentaire.

Notre travail n'est pas facilité par le contexte actuel où, il faut l'avouer, la gestion des dossiers inactifs ne semble pas prioritaire pour certains gestionnaires. Beaucoup de questions surgissent: les locaux pour ranger les dossiers inactifs sont-ils disponibles et adéquatement aménagés? Quelle direction assume les coûts associés au déménagement et au matériel? Quels seront les espaces alloués aux archives dites médicales vis-à-vis des archives administratives? Malgré ces interrogations, l'équipe de gestion documentaire ne perd pas de vue ses objectifs en regard de la gestion des espaces nécessaires à une gestion efficace des documents actifs, semi-actifs et inactifs. Notre insistance à obtenir des réponses a donné des résultats puisque les derniers développements de décembre 2016 laissent entrevoir la possibilité de mettre sur pied un dépôt d'archives historiques permanent dans le secteur Saguenay et un autre dans le secteur Lac-Saint-Jean. L'avenir nous dira si cela se concrétisera.

\subsubsection{La prise en charge des documents en format numérique}

Bien que l'essentiel de nos actions aient porté jusqu'à présent sur la prise en charge des documents en format papier, nous étions néanmoins conscients, dès le départ, de l'importance de tenir compte des documents numériques. Ainsi, en avril 2016, un projet de stratégie de prise en charge des documents numériques actifs a résulté de nos premières discussions. Ce projet pourrait vraisemblablement avancer puisqu'une de nos prochaines actions en 2017 consistera à rencontrer les agentes administratives des directions afin de connaître, entre autres, leurs besoins 
particuliers en ce qui concerne les documents actifs en format numérique. On ne peut ignorer l'importance de l'adhésion de cette catégorie de professionnels, producteurs/créateurs de documents papier et numérique, dans le succès d'un SGID. Dans la même veine, nous avons commencé au même moment une démarche en vue de préserver les sites Web et préparé, en juin 2016, un projet de sauvegarde des archives historiques numériques des établissements fusionnés. Finalement, en novembre 2016, quelques vidéos ayant une valeur historique sur support VHS furent transférées sur disques compacts (CD). Ces quelques réalisations constitueront certes des acquis sur le plan de la prise en charge concrète des documents numériques le moment venu.

\section{BILAN À CE JOUR: FACTEURS POSITIFS ET ÉCUEILS}

Pouvons-nous cibler avec certitude un élément déclencheur de la prise en charge de la gestion des documents administratifs au sein du CIUSSS-SLSJ ? Notre initiative personnelle? Les écrits du CNASSS ? Chose certaine, en opérant un certain recul, il appert que certains facteurs ont contribué à initier cette prise en charge. Notons, entre autres, I'inscription de l'activité « gestion documentaire » dans l'organigramme; la nomination d'un gestionnaire à titre de responsable de cette activité et de l'équipe de gestion documentaire; la participation de ce gestionnaire dans la recherche de solutions auprès de la direction et de ses collègues gestionnaires ${ }^{15}$; la mise sur pied d'une véritable équipe de gestion documentaire; la motivation et le professionnalisme de chaque membre de cette équipe; le respect des opinions de chaque membre de cette équipe, malgré certaines discussions un tantinet houleuses mais combien productives; la nécessaire collaboration avec le service informatique et celui des archives médicales.

Bien sûr, tout n'est pas rose! Pensons aux difficultés rencontrées pour dénicher, auprès des gestionnaires concernés, des locaux pouvant recevoir les documents semi-actifs et inactifs (archives historiques), à ce combat toujours d'actualité dans le réseau de la santé afin de faire reconnaître notre profession et notre champ d'activité professionnelle comme distinct de celui des archives médicales ${ }^{16}$, etc. En ce qui regarde le rythme de déploiement du SGID, les actions de l'équipe de gestion documentaire à ce jour peuvent sembler de prime abord tâtonnantes. Cependant, malgré la réalité inhérente à la période actuelle de transition, 
les éléments de base pour l'implantation d'un véritable SGID se mettent graduellement en place.

Somme toute, les défis sont importants et la route pourrait s'avérer relativement longue et parsemée d'embûches avant que le CIUSSS-SLSJ ne puisse compter sur un SGID organisationnel et totalement fonctionnel. Toutefois, l'avenir s'annonce prometteur et dépendra en grande partie des ressources humaines, financières et matérielles disponibles. Dans cet ordre d'idées, il est à espérer que l'importance de la gestion des documents administratifs et celle du rôle de l'équipe de gestion documentaire soient non seulement maintenues, mais renforcées. En contrepartie, les autorités du CIUSSS-SLSJ peuvent compter sur une équipe de gestion documentaire dont les membres sont passionnés par leur métier et motivés par le défi qui se présente à eux.

Dany Ouellet

\section{NOTES}

1. Ces établissements sont: I'Agence de la santé et des services sociaux du SaguenayLac-Saint-Jean; le Centre de réadaptation en déficience intellectuelle et en troubles envahissants du développement du Saguenay-Lac-Saint-Jean; le Centre de santé et de services sociaux Cléophas-Claveau; le Centre de santé et de services sociaux de Chicoutimi; le Centre de santé et de services sociaux de Jonquière; le Centre de santé et de services sociaux de Lac-Saint-Jean-Est; le Centre de santé et de services sociaux Domaine-du-Roy; le Centre de santé et de services sociaux Maria-Chapdelaine; le Centre jeunesse du Saguenay-Lac-Saint-Jean.

2. Site Web consulté le 7 décembre 2016: http://santesaglac.com/ciusss (lien non fonctionnel).

3. Les opinions émises dans cet article n'engagent que l'auteur.

4. Bibliothèque et Archives nationales du Québec (BAnQ), I'Association québécoise des établissements de santé et de services sociaux (AQESSS), I'Association des archivistes du Québec (AAQ), I'Association sur l'accès et la protection de l'information (AAPI), I'Association des gestionnaires de l'information de la santé du Québec (AGISQ) et aux établissements universitaires offrant des formations en gestion des documents administratifs.

5. Le CNASSS est né en 2015 suite à l'abolition de I'AQESSS (Association québécoise des établissements de santé et de services sociaux) qui était chargé auparavant d'élaborer le recueil des règles de conservation du réseau de la santé. Ce comité, sous 
la supervision de la Direction générale des Archives nationales de BAnQ, assume donc maintenant le leadership dans ce dossier. Afin d'assurer une meilleure représentativité et une meilleure connaissance des réalités de ce milieu, le comité peut compter sur une étroite collaboration de ce dernier. De fait, chaque secteur de la santé et des services sociaux est représenté au sein du comité. Outre les activités reliées au recueil, le comité a aussi pour mission d'élaborer des procédures pour rappeler aux organismes de ce secteur public leurs responsabilités par rapport à la Loi sur les archives. Notons enfin que le CNASSS n'est pas un organisme légalement constitué, mais demeure cependant un comité permanent.

6. Ce document a été mis à jour en juin 2015 suite à l'adoption de la loi.

7. Le comité de la haute direction du CIUSSS-SLSJ est l'équivalent d'un comité exécutif.

8. Le contenu de ce guide est constitué d'une série de questions portant sur l'évaluation sommaire de l'usage des outils de gestion des documents administratifs (la politique de gestion des documents administratifs, le plan de classification, le calendrier de conservation, les outils électroniques de repérage, le guide de gestion des documents administratifs, la protection des documents essentiels, les documents confidentiels, le mode de destruction des documents confidentiels et la gestion des documents numériques), suivi d'une évaluation sommaire de la masse documentaire et de commentaires des employés concernés. Le tout est complété par les observations in situ par l'équipe de gestion documentaire et de quelques recommandations.

9. Constellio, Documentik, Gestion virtuelle, Logiplus et SyGED.

10. Notre évaluation de la masse documentaire ne portait que sur les documents administratifs et excluait les documents à caractère clinique communément appelés "archives médicales». Notre équipe de gestion documentaire a décidé de distinguer ces deux secteurs d'activités en gestion de l'information parce que leurs assises légales sont différentes, leurs règles de fonctionnement et les outils utilisés sont particuliers à chacun. De fait, les archives médicales prennent en charge spécifiquement les dossiers de santé et autres documents relatifs aux services de santé, sociaux et communautaires dispensés aux usagers du réseau québécois de santé et de services sociaux. Certes, la gestion des documents administratifs et la gestion des archives médicales sont bien présentes dans nos établissements de santé, mais ce sont deux systèmes tout à fait différents. D'ailleurs, historiquement, la gestion des archives médicales est sous la responsabilité d'un service structuré et reconnu dans l'établissement. Ce dernier a un mandat clairement défini et il peut compter sur des ressources humaines, matérielles et financières qui lui sont propres. Pour la gestion des documents administratifs cependant, c'est en général malheureusement fort différent. II n'existe que peu ou pas de service de gestion documentaire à proprement parler.

11. Le système de gestion des documents de référence et des documents audiovisuels relatifs à la bibliothéconomie, le système de gestion des archives médicales et les documents personnels à un employé ne sont pas directement concernés par la présente politique. 
12. À cette fin, l'équipe de gestion documentaire participe, entre autres, à toute activité relative à l'introduction d'une nouvelle technologie d'information dont l'objet est le traitement de données et de documents soumis au cadre légal en la matière.

13. GALA (Gestion de l'application de la Loi sur les archives), lancé officiellement en février 2014, rend transactionnel le processus de création, de soumission, d'analyse et d'approbation des calendriers de conservation entre les organismes publics et BAnQ.

14. En novembre 2016, le Secrétariat du Conseil du trésor du gouvernement du Québec faisait parvenir, à l'ensemble des dirigeants sectoriels de l'information du réseau de la santé et des services sociaux, une lettre indiquant que les organismes publics pourraient considérer et favoriser la sélection d'un logiciel libre pour la gestion intégrée des documents. Un produit a ainsi été sélectionné dans le cadre de la mesure 19 de la Stratégie gouvernementale en TI: Rénover l'État par les technologies de l'information. Au cours des prochains mois, nous devrions donc être en mesure de savoir si cette orientation doit être considérée comme une recommandation, une «forte » recommandation ou une obligation.

15. À notre avis, encore plus que ne l'est l'inscription de l'activité «gestion documentaire» dans l'organigramme, faire comprendre et accepter la nécessité et les avantages d'implanter un SGID auprès des décideurs et des gestionnaires représente un défi important pour l'équipe de gestion documentaire.

16. Force est de constater (voir en bibliographie l'enquête de 2006) que la culture organisationnelle des établissements de santé au Québec en matière de gestion de l'information documentaire est très fortement associée à la gestion du dossier de santé (archives médicales). La gestion des documents administratifs est malheureusement souvent laissée pour compte.

\section{BIBLIOGRAPHIE}

Bibliothèque et Archives nationales du Québec (BAnQ). Direction des services aux usagers et aux partenaires. (2015, mai). Création du CIUSSS et calendriers de conservation. Directive interne. Québec, Québec: BAnQ.

BOUCHARD, C., Gendron, M. et Ducasse, Y. (1992). Les archives médicales et administratives dans les établissements de santé du Québec. Deux services parallèles? Archives 24 (1-2), 263-272.

Centre intégré universitaire de la santé et des services sociaux (CIUSSS) du Saguenay-Lac-Saint-Jean. Équipe de gestion documentaire. 
(2016, octobre). État de situation de la gestion documentaire des 9 établissements fusionnés. Rapport interne. Chicoutimi, Québec: CIUSS.

Centre intégré universitaire de la santé et des services sociaux (CIUSSS) du Saguenay-Lac-Saint-Jean. Équipe de gestion documentaire. (2015, décembre). Le portrait documentaire des établissements fusionnés. Prise d'inventaire. Rapport interne. Chicoutimi, Québec: CIUSS.

Centre intégré universitaire de la santé et des services sociaux (CIUSSS) du Saguenay-Lac-Saint-Jean. (2016, juin). Politique relative à la gestion des documents administratifs. PO-AJ.011. Chicoutimi, Québec: CIUSS. Repéré à http://santesaglac.gouv.qc.ca/medias/documents/politiques internes/politique relative gestion documents administratifs vf 201701-19.pdf

Comité national des archives de la santé et des services sociaux (CNASSS). (2015, juin). Les documents inactifs du réseau de la santé et des services sociaux dans le contexte de la Loi modifiant l'organisation et la gouvernance du réseau de la santé et des services sociaux notamment par l'abolition des agences régionales. Principes directeurs. Montréal, Québec: Bibliothèque et Archives nationales du Québec. Repéré à http://www.banq.qc.ca/documents/archives/archivistique gestion/ partenaires/organismes publics/fusion/Documents inactifs sante loi_10 2015-06-05.pdf

Comité national des archives de la santé et des services sociaux (CNASSS). (2017). Recueil des règles de conservation des documents des établissements de santé et de services sociaux du Québec. Québec, Québec: Bibliothèque et Archives nationales du Québec. Repéré à http://www.banq.qc.ca/dotAsset/ae7e54d4-6379-4fea-88d8bdac78173e06.pdf

Loi modifiant l'organisation et la gouvernance du réseau de la santé et des services sociaux notamment par l'abolition des agences régionales. (RLRQ, chapitre 0-7.2.). Repéré à http://www.legisquebec.gouv.qc.cal fr/ShowDoc/cs/O-7.2

Loi sur l'accès aux documents des organismes publics et sur la protection des renseignements personnels. (RLRQ, chapitre A-2.1.) Repéré à http:// legisquebec.gouv.qc.ca/fr/ShowDoc/cs/A-2.1 
Loi sur les archives. (RLRQ, chapitre A-21.1.) Repéré à http://www. legisquebec.gouv.qc.ca/fr/showdoc/cs/A-21.1

OUELLET, D. (2006, décembre). Enquête sur la gestion documentaire dans les centres de santé et de services sociaux du Québec. Document interne. Chicoutimi, Québec.

OUELLET, D. (2015, mai). La prise en charge de la gestion documentaire au Centre universitaire de santé et des services sociaux (CIUSSS) du Saguenay-Lac-Saint-Jean: une nécessité. Document interne. Chicoutimi, Québec.

OUELLET, D. (2014, novembre). La réforme de notre système de santé et la gestion de l'information documentaire. Document interne. Chicoutimi, Québec.

OUELLET, D. - Consuldoc enr. (2016, novembre). Guide pour élaborer le portrait documentaire d'un poste de classement des documents administratifs. Document interne. Chicoutimi, Québec: Consuldoc Enr. 